\title{
NASA Global Flood Mapping System
}

Fritz Policelli, NASA GSFC

Dan Slayback, SSAI/ GSFC

Bob Brakenridge, University of Colorado Joe Nigro, SSAI/ GSFC

Alfred Hubbard, SSAI/GSFC

September „, 2017 


\section{Selection of users}

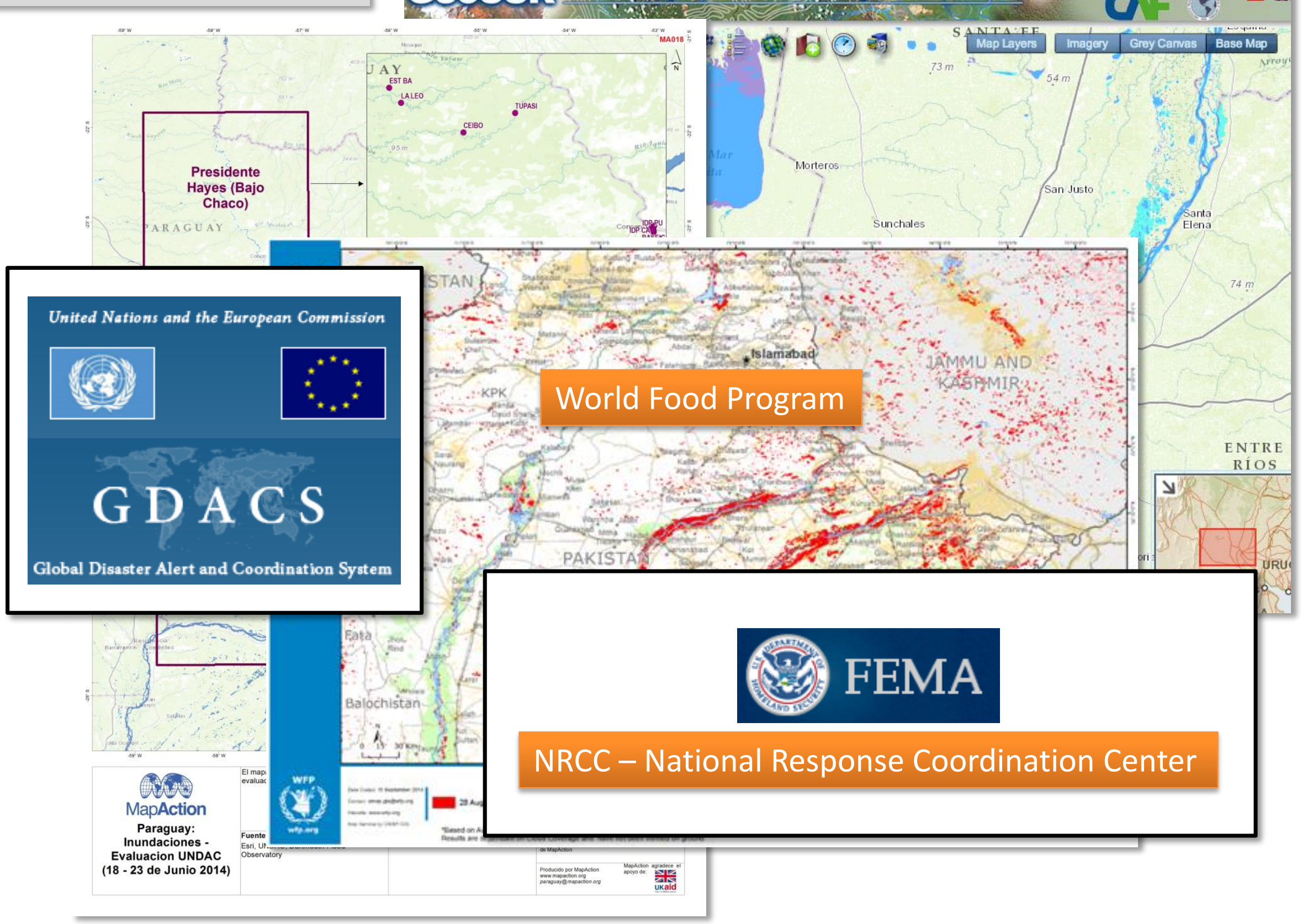




\section{Product utility - key factors}

- Near real time, automated production

- Flood spatial extent

- Cloudiness

- Pixel resolution: $250 m$

- Flood temporal extent

- Flash floods / short duration on ground?

- Landcover

- Water under vegetation cover vs open water 


\section{A little history}

- Bob Brakenridge (Dartmouth Flood Observatory) manually generated flood maps using MODIS rapid response imagery

- Product distribution via large-format digital maps (tif and pdf)

- Useful product, but:

- Generated from rapid response jpegs not meant for analysis

- Not automated

- Not easily incorporated into GIS

- NASA funded GSFC to build an automated daily, global, near real-time system

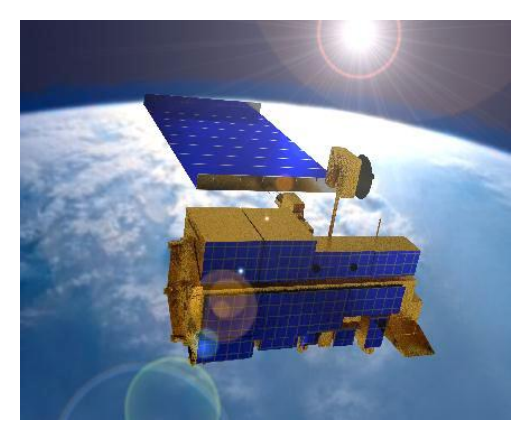

Terra
The MODIS sensor is on both the NASA Terra and Aqua satellites

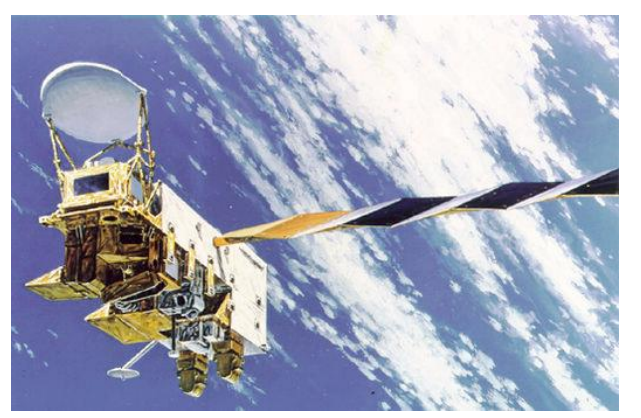

Aqua 
MODIS product distribution system: http://oas.gsfc.nasa.gov/floodmap

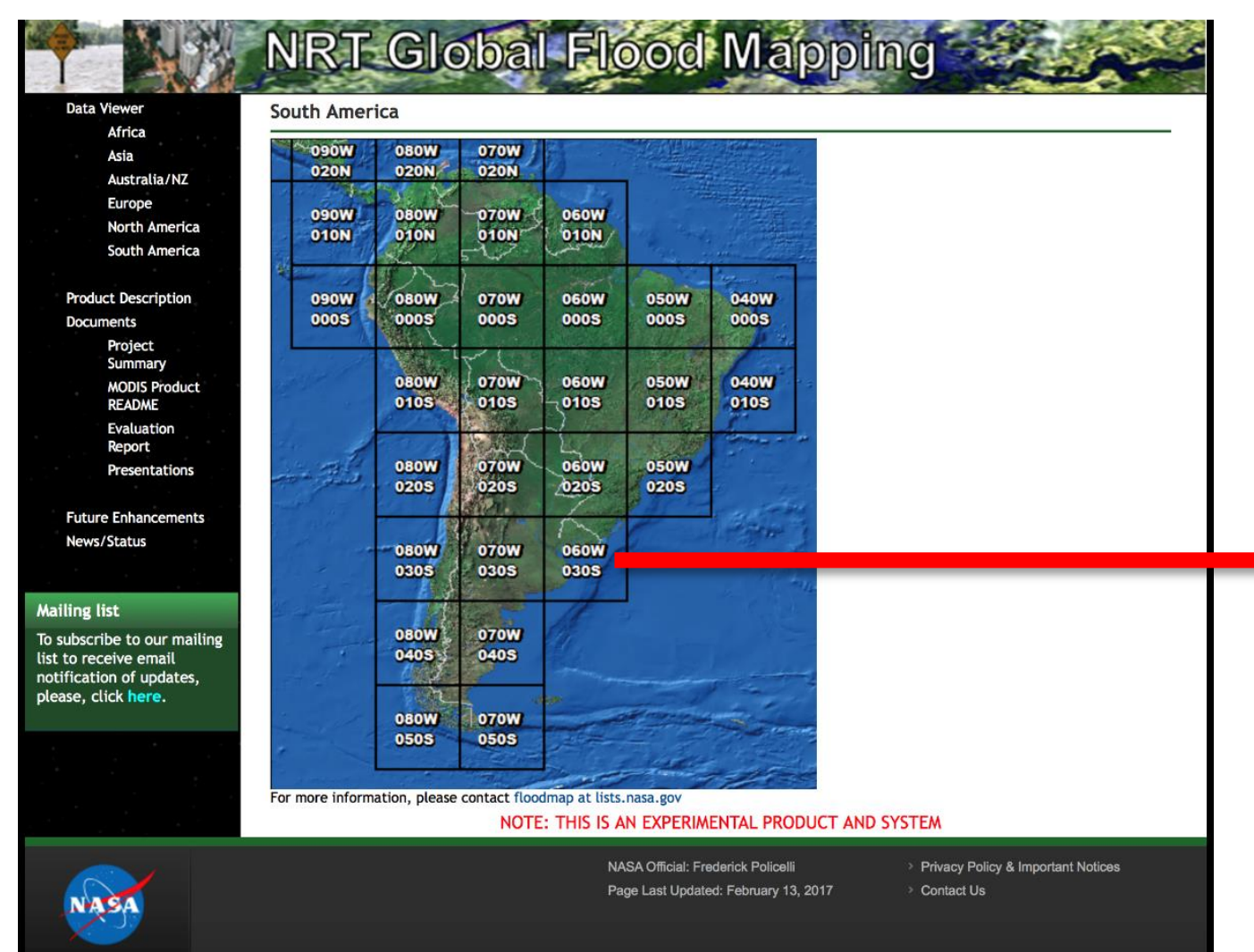

Continental tile index

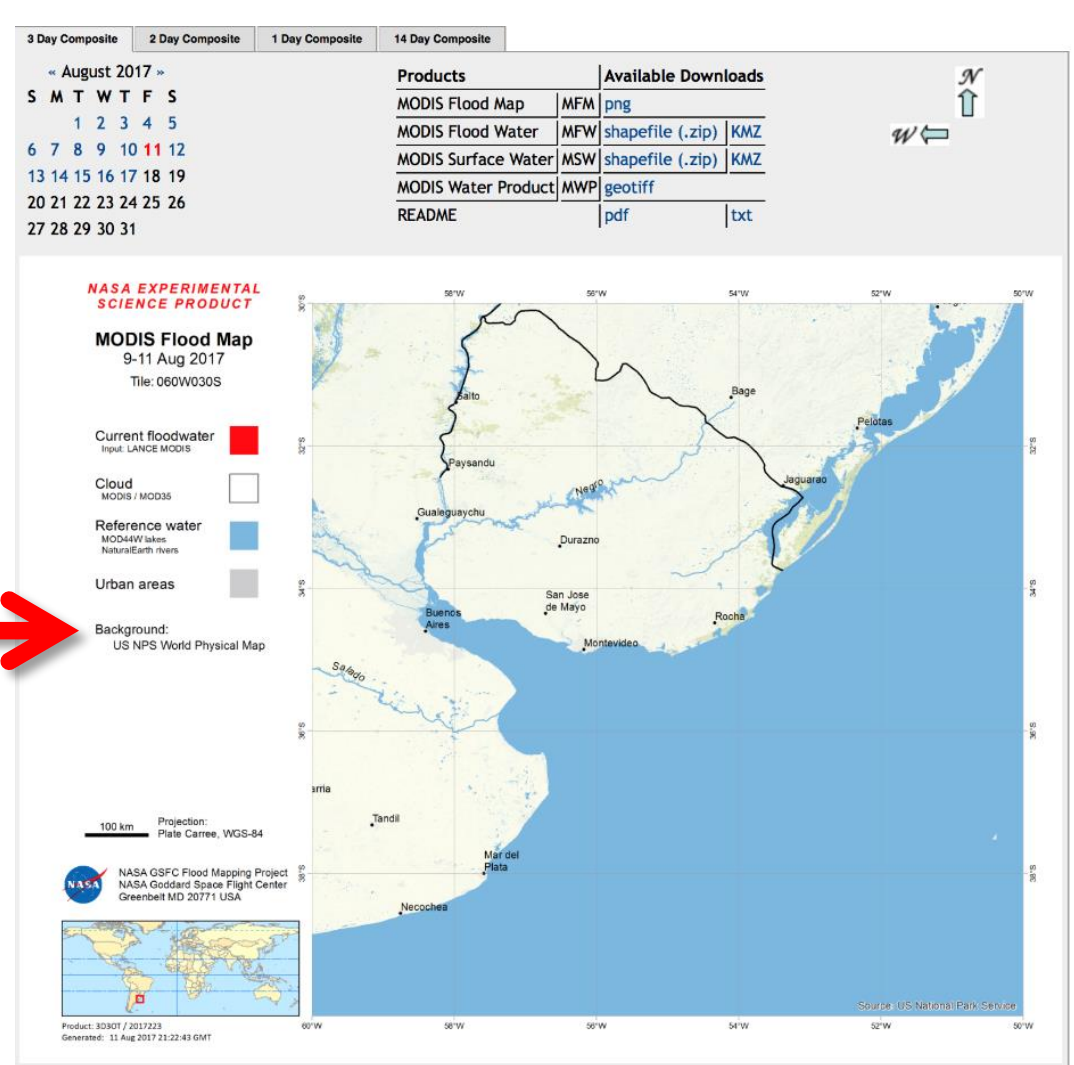

Specific tile

- Date selector

- Available product/format downloads 


\section{MODIS Flood Product}

Input data: near real-time MODIS imagery from the LANCE system at NASA Goddard Space Flight Center

- Daily calibrated Terra and Aqua MODIS reflectances for bands 1, 2,7

- Corresponding cloud products for cloud and cloud shadow masking

- Delivered in 10 deg. $X 10$ deg. tiles 


\section{Water detection algorithm}

Water if: $\quad \frac{(\text { Band } 2+A)}{(B \text { Band } 1+B)}<C$

$$
\begin{aligned}
& \text { AND } \quad \text { Band } 1<D \\
& \text { AND } \quad \text { Band } 7<E
\end{aligned}
$$

\begin{tabular}{l|l}
$A$ & 13.5 \\
\hline$B$ & 1081 \\
\hline$C$ & 0.7 \\
\hline$D$ & 2027 \\
\hline$E$ & 676
\end{tabular}

- Bands are MOD09 surface reflectance product

- Developed by Bob Brakenridge, Dartmouth Flood Observatory, U. Colorado 


\section{Additional Processing}

- Multi-look compositing: require multiple positive water detections to label a pixel as water - minimizes cloud shadow false-positives

- Terrain shadows masked using DEM and solar geometry

- Flood: water exceeding normal surface water, as defined by static global water map (MOD44W) 


\section{Automated MODIS Flood Map Production System}

- Fully automated (since Nov 2011)

- $22310 \times 10^{\circ}$ tiles $\times 3$ products (2-day, 3 -day, 14 -day) $=669$ daily product suite generated

- Product suite includes: geotiffs, shapefiles, KML (Google Earth), and graphic maps (png)

- Products typically available within 6 hours of Aqua overpass ( 8:00 PM local time)

- Delivery via web download 


\section{Products: 3 elements}

1. Composite period (balance between currency and spatial completeness):

- Standard products: 2-day, 3-day

- Short-term: 1-day

- Extended: 14-day

2. Product name:

- MWP: MODIS Water Product (core product)

- MFW: MODIS Flood Water (derived)

- MSW: MODIS Surface Water (derived)

- MFM: MODIS Flood Map (derived)

3. Formats:

- Raster / geotiff (some products)

- Vector / shapefile \& KML (some products)

- Graphic product/ png 


\section{MODIS Flood Map Compositing}

- 1-day composite: requires 1 water observation over current day's imagery (potentially 2 observations with Terra and Aqua). Not normally generated.

- 2-day: requires 2 water observations over 2 days of imagery (potentially 4 observations).

- 3-day: requires 3 water observations over 3 days of imagery (potentially 6 observations).

- 14-day: second order composite, combining the 14 previous 3-day products. Provides a recent-historical view. 
Distribution via NASA website: http://oas.gsfc.nasa.gov/floodmap

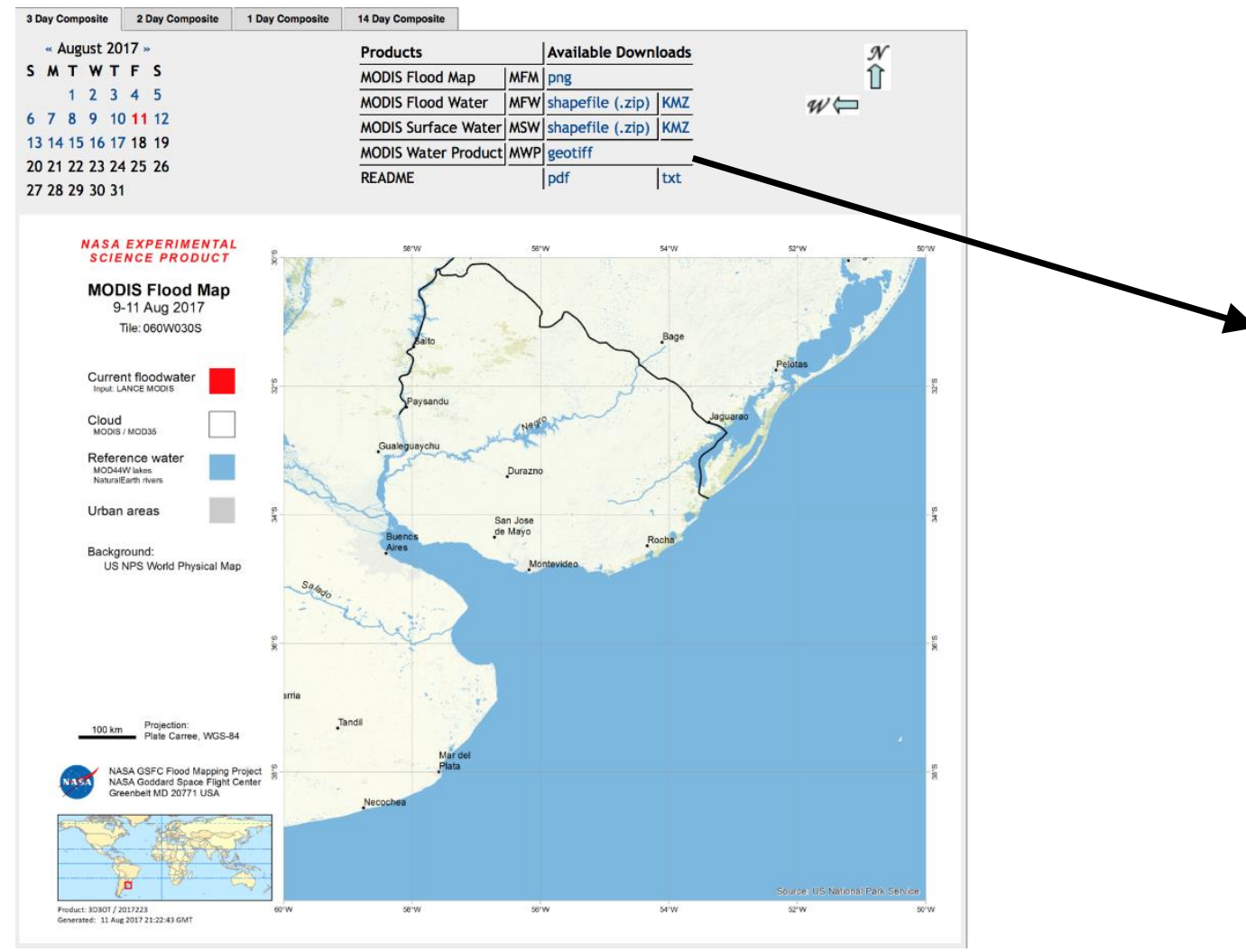

\begin{tabular}{l|l|l|l} 
Products & \multicolumn{2}{|l}{ Available Downloads } \\
\hline MODIS Flood Map & MFM & png \\
\hline MODIS Flood Water & MFW & shapefile (.zip) & KMZ \\
\hline MODIS Surface Water & MSW & shapefile (zip) & KMZ \\
\hline MODIS Water Product & MWP & geotiff & \\
\hline README & pdf & txt
\end{tabular}

Product downloads table

\section{W030S}

- date selector

- product/format downloads

- navigation tool 


\section{Products: MODIS Water Product (MWP)}

- Core product

- Geotiff format

- Values:

0 : Insufficient data (for composite period)

1: No water detected

2: Surface water (corresponding to Reference water pixels).

3: Flood water (water outside Reference water pixels).

- Coastal strip visible; ocean water removed beyond $10 \mathrm{~km}$

- MOD35 Cloud used only to populate "Insufficient data"; water detected through cloud IS reported

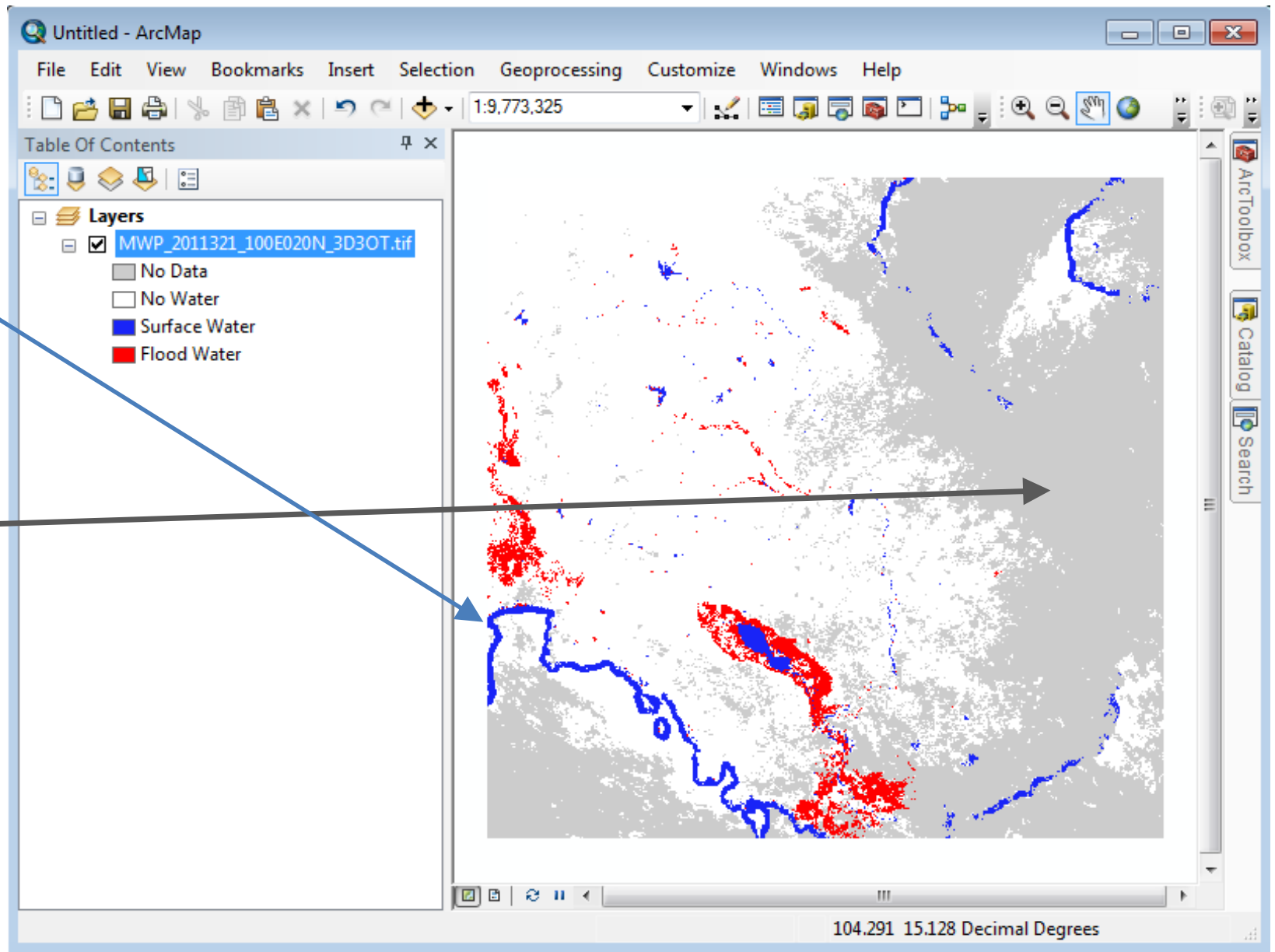




\section{Products: MODIS Flood Map (MFM) $10^{\circ}$ tile graphic map (PNG)}

NASA EXPERIMENTAL
SCIENCE PRODUCT

MODIS Flood Map

1-2 Nov 2011

Tile: $100 \mathrm{E} 020 \mathrm{~N}$

Current floodwater

Input: LANCE MODIS

Cloud

MODIS / MOD35

Reference water

MOD44W lakes

NaturalEarth rivers

Urban areas

Background:

US NPS World Physical Map

$100 \mathrm{~km}$

Projection:

Plate Carree, WGS-84

Office of Applied Sciences NASA Goddard Space Flight Center Greenbelt MD 20771 USA

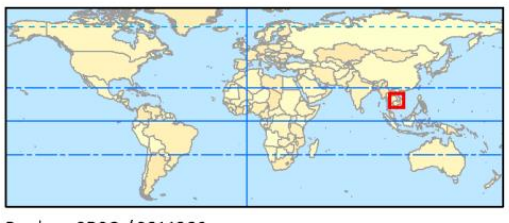

Product: $2020 / 2011306$

Generated: 15 Feb 2012 18:50:08 GMT

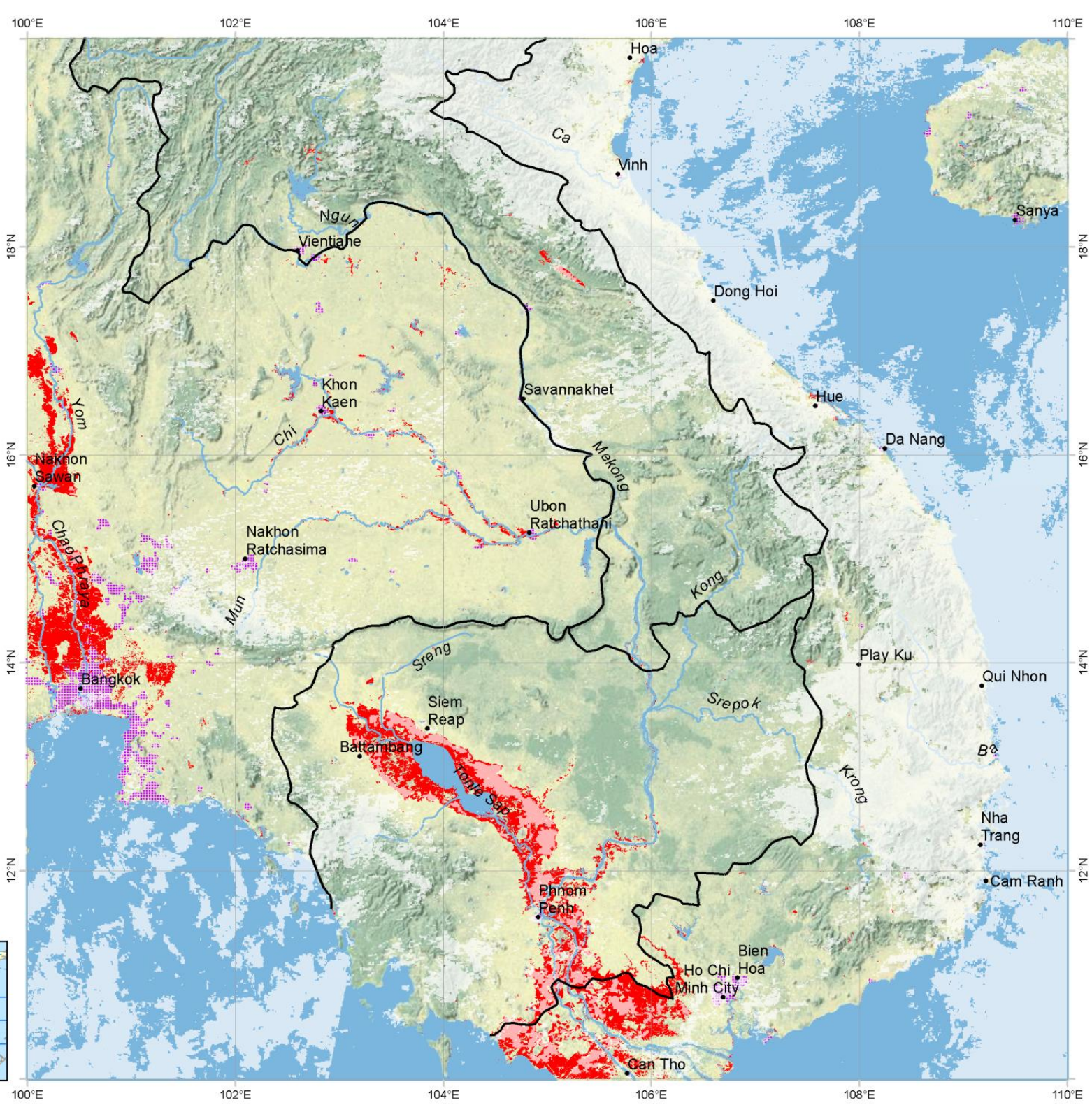




\section{Products: MODIS Surface \& Flood Water (MSW, MFW) shapefiles}

- Vectorized from MWP (raster) product

- Does not indicate where insufficient data to determine (value 0 of MWP product)

- Provides area and centroid per polygon

- Production can fail if too many polygons

- KML production skipped if \#polygons $>15000$

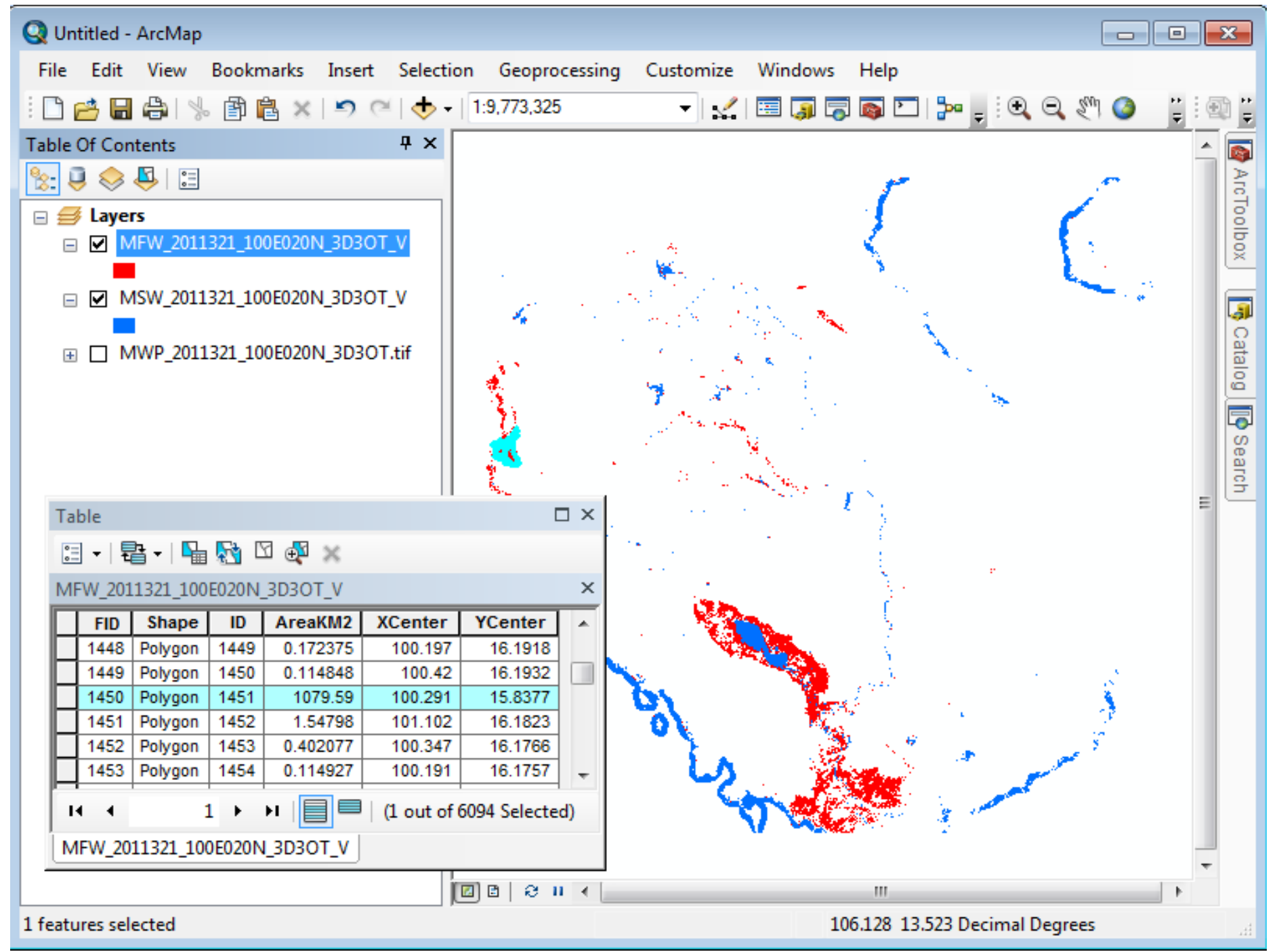




\section{Products: MODIS Surface \& Flood Water KML files (Google Earth)}

KML files in Google Earth:

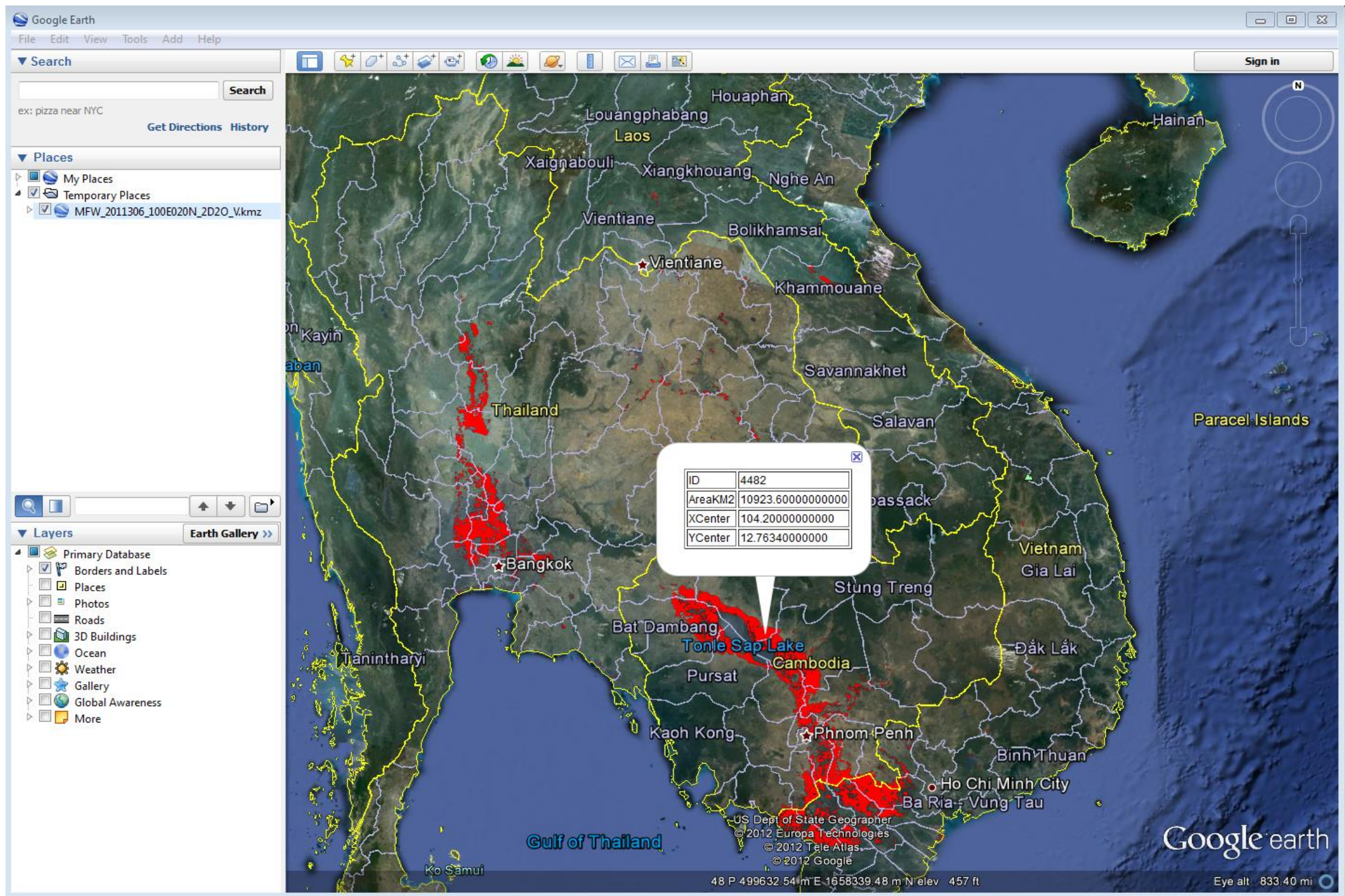


Products: MODIS Surface \& Flood Water KML files (Google Earth)

Google Earth zoomed in -- Bangkok's Don Muang Airport runways under water:

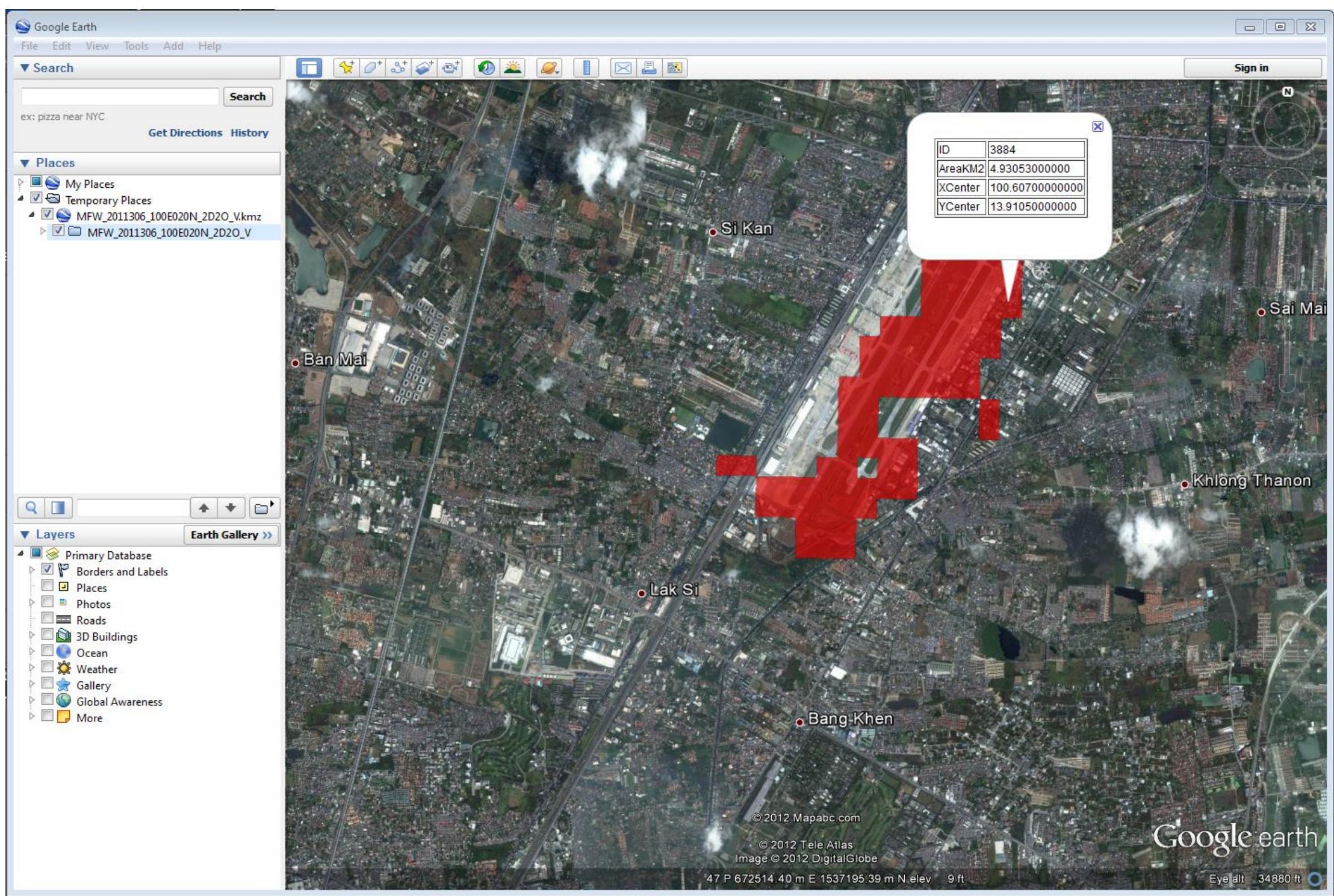




\section{MODIS Flood Product Evaluation}

Purpose:

- Is water detection algorithm working

- correctly detecting visually obvious water?

- Are certain situations problematic?

- Are the multi-day composited products working well?

- Differences between detection of flood water vs normal water

Evaluation method:

- Globally distributed flood and permanent water sites ( 50 each)

- Visual and qualitative assessment of performance

- raw MODIS and Landsat imagery used to help inform assessment 

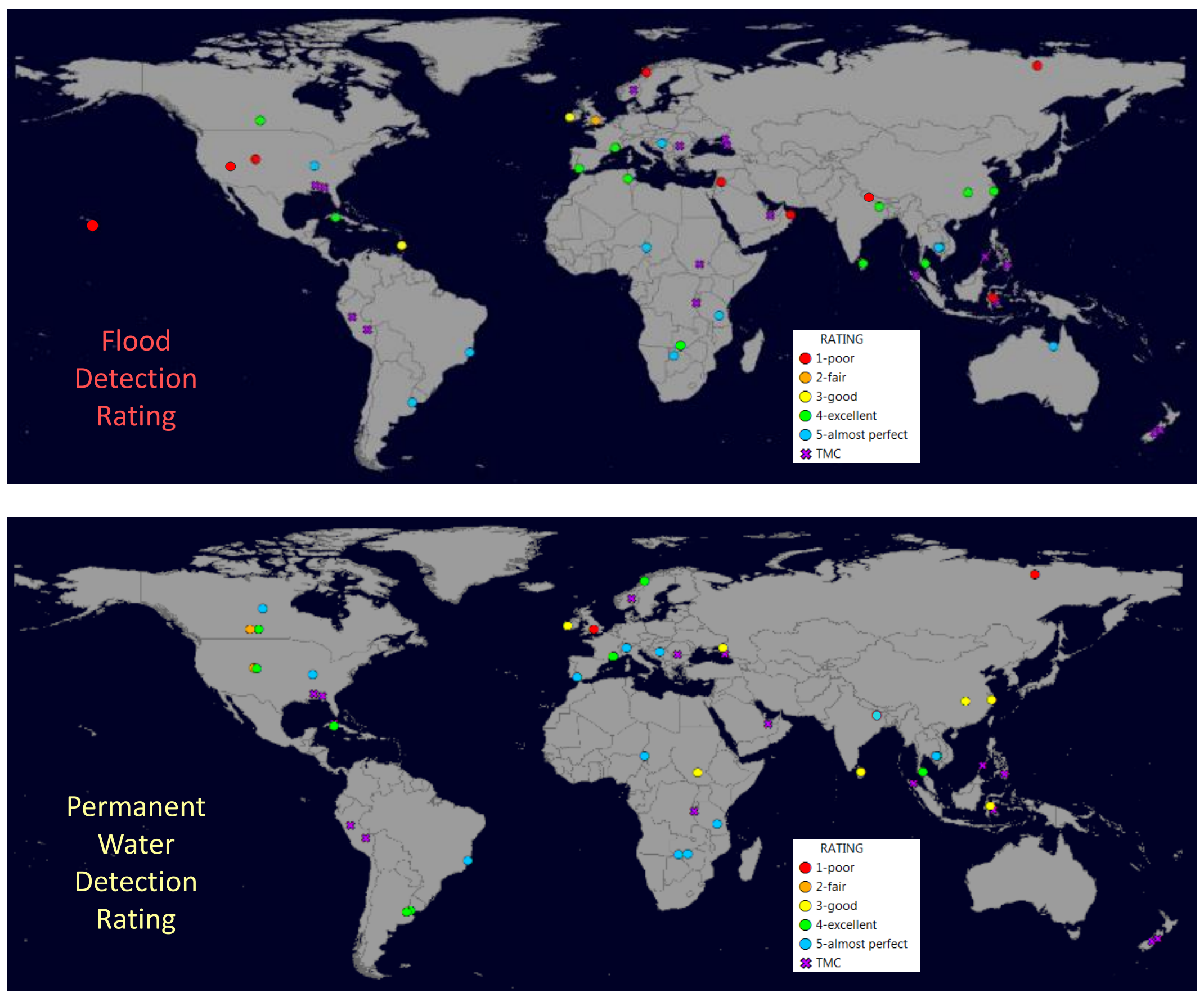


\section{Flood Detection Ratings}

\begin{tabular}{|c|c|c|}
\hline RATING & Count & $\%$ \\
\hline 5-almost perfect & 11 & 21 \\
\hline 4-excellent & 10 & 19 \\
\hline 3-good & 2 & 4 \\
\hline 2-fair & 1 & 2 \\
\hline 1-poor & 11 & 21 \\
\hline TMC - too many clouds & 17 & 33 \\
\hline Outside product coverage area & 1 & Eliminated from equation \\
\hline TOTALS & 53 & 100 \\
\hline
\end{tabular}

\section{Permanent Water Detection Ratings}

\begin{tabular}{|c|c|c|}
\hline RATING & Count & $\%$ \\
\hline 5-almost perfect & 15 & 28 \\
\hline 4-excellent & 9 & 17 \\
\hline 3-good & 7 & 13 \\
\hline 2-fair & 2 & 4 \\
\hline 1-poor & 4 & 8 \\
\hline TMC - too many clouds & 16 & 30 \\
\hline Outside product coverage area & 1 & Eliminated from equation \\
\hline TOTALS & 54 & 100 \\
\hline
\end{tabular}


Correct flood and permanent water identification

Brazil: 02 January 2014

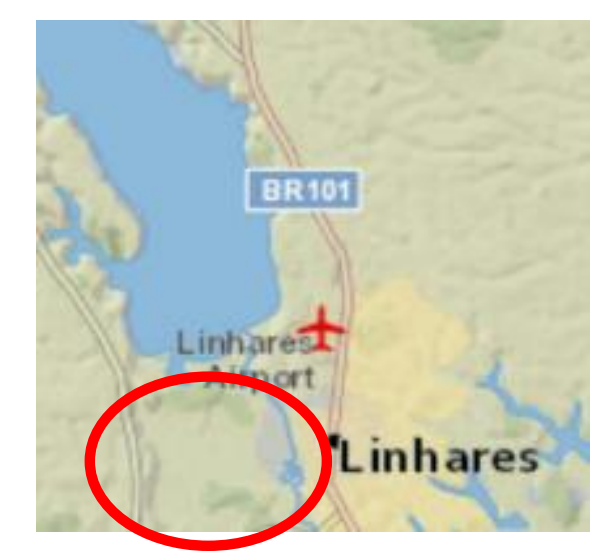

National Geographic base map

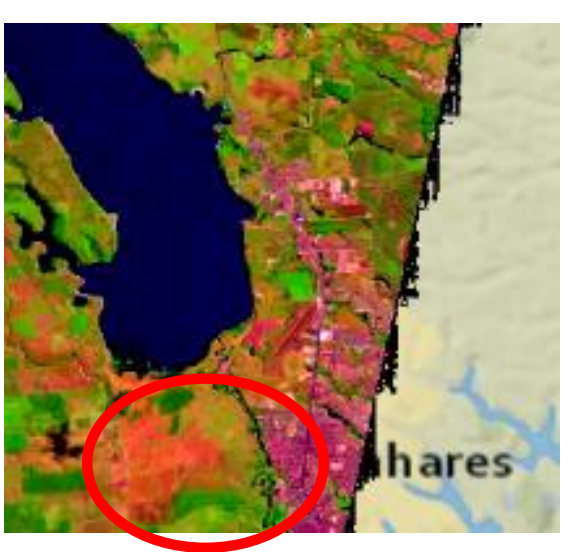

Landsat 8 Pre-flood Apr 21, 2013

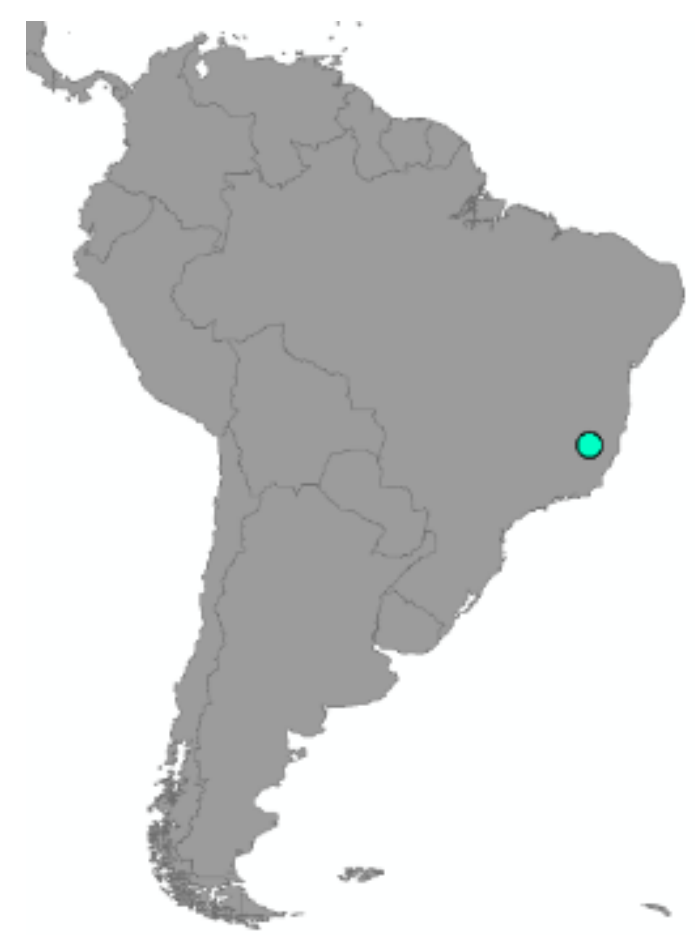

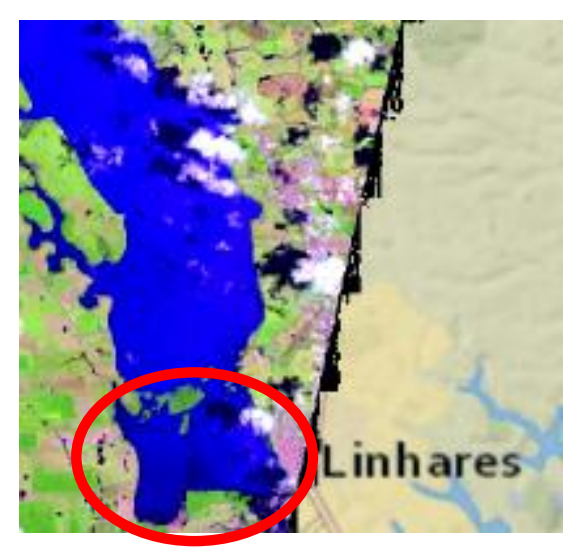

Landsat 8 Flood Jan 2, 2014

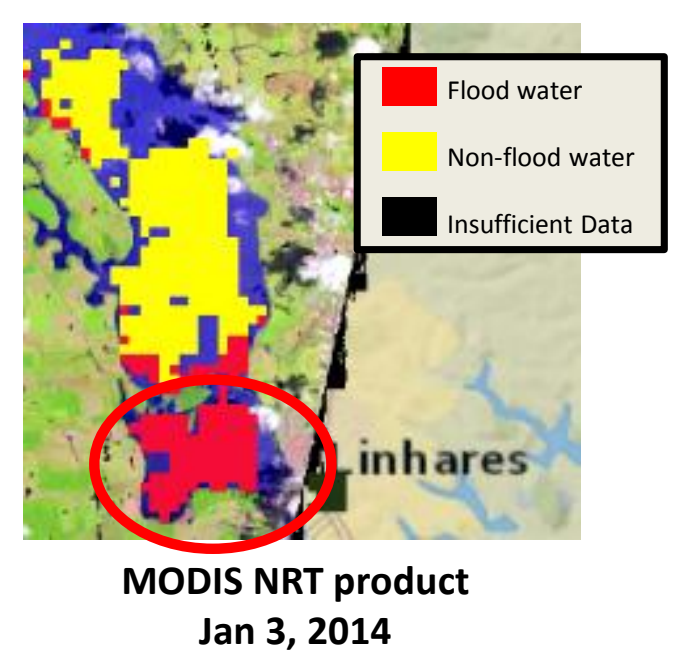




\section{Example: Correct flood identification}

Kentucky: 04 Jan 2014

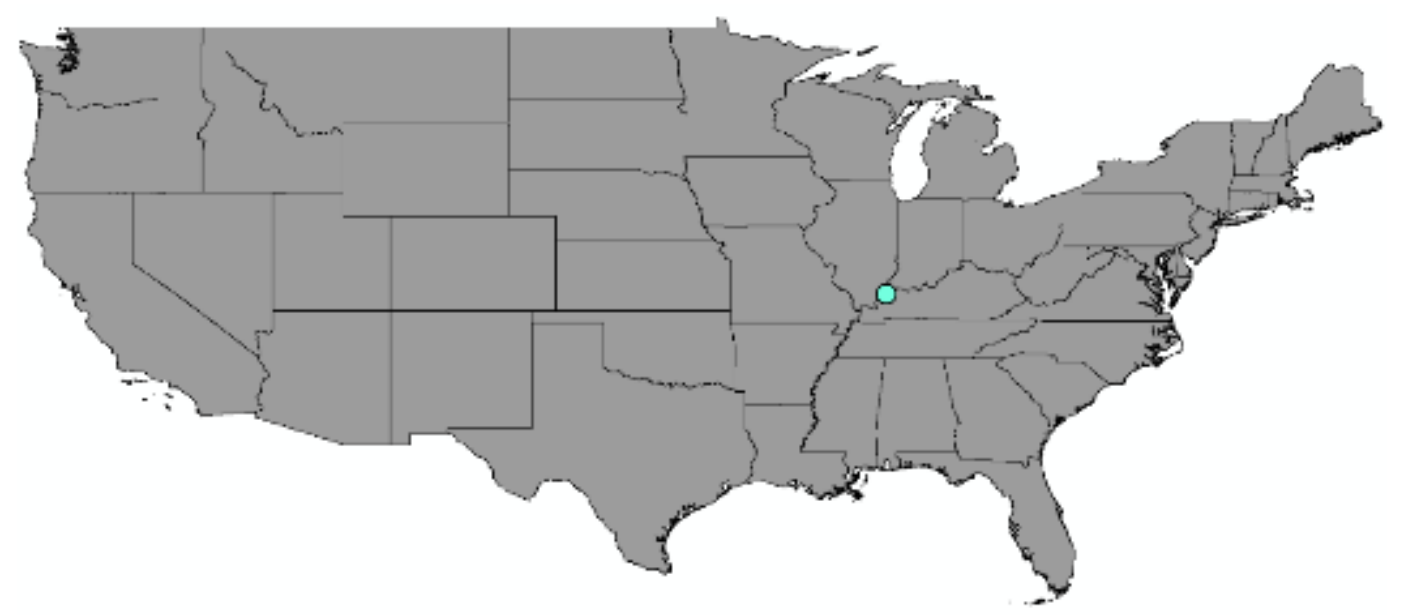

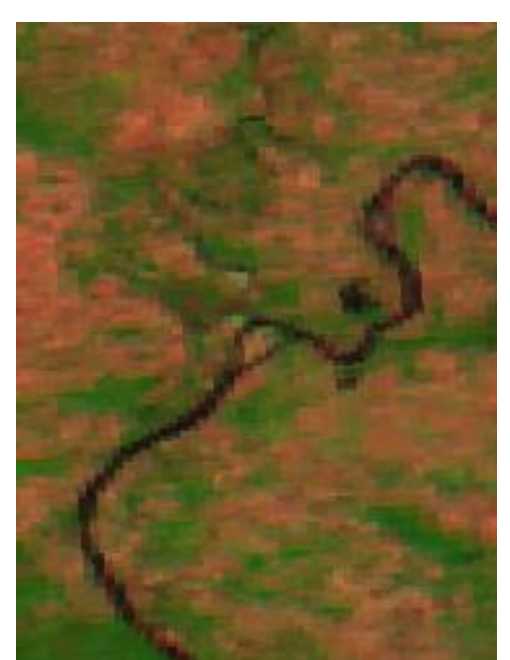

MODIS (MOD09) Pre-Flood Oct 12, 2013

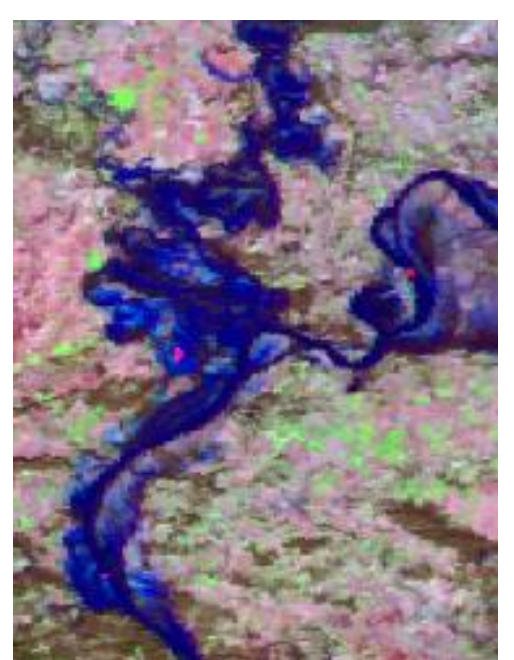

MODIS (MOD09) Flood Jan 4, 2014

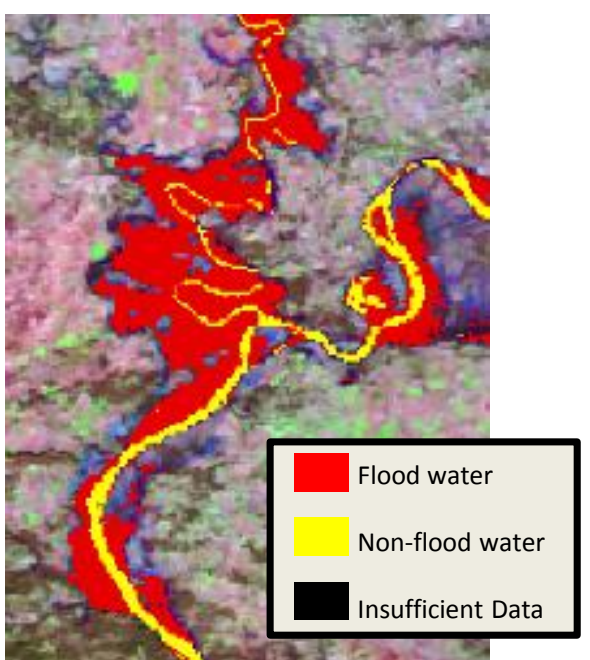

MODIS NRT Product Jan 4, 2014 


\section{Terrain shadow false-positives}

OMAN: mid November 2013 products
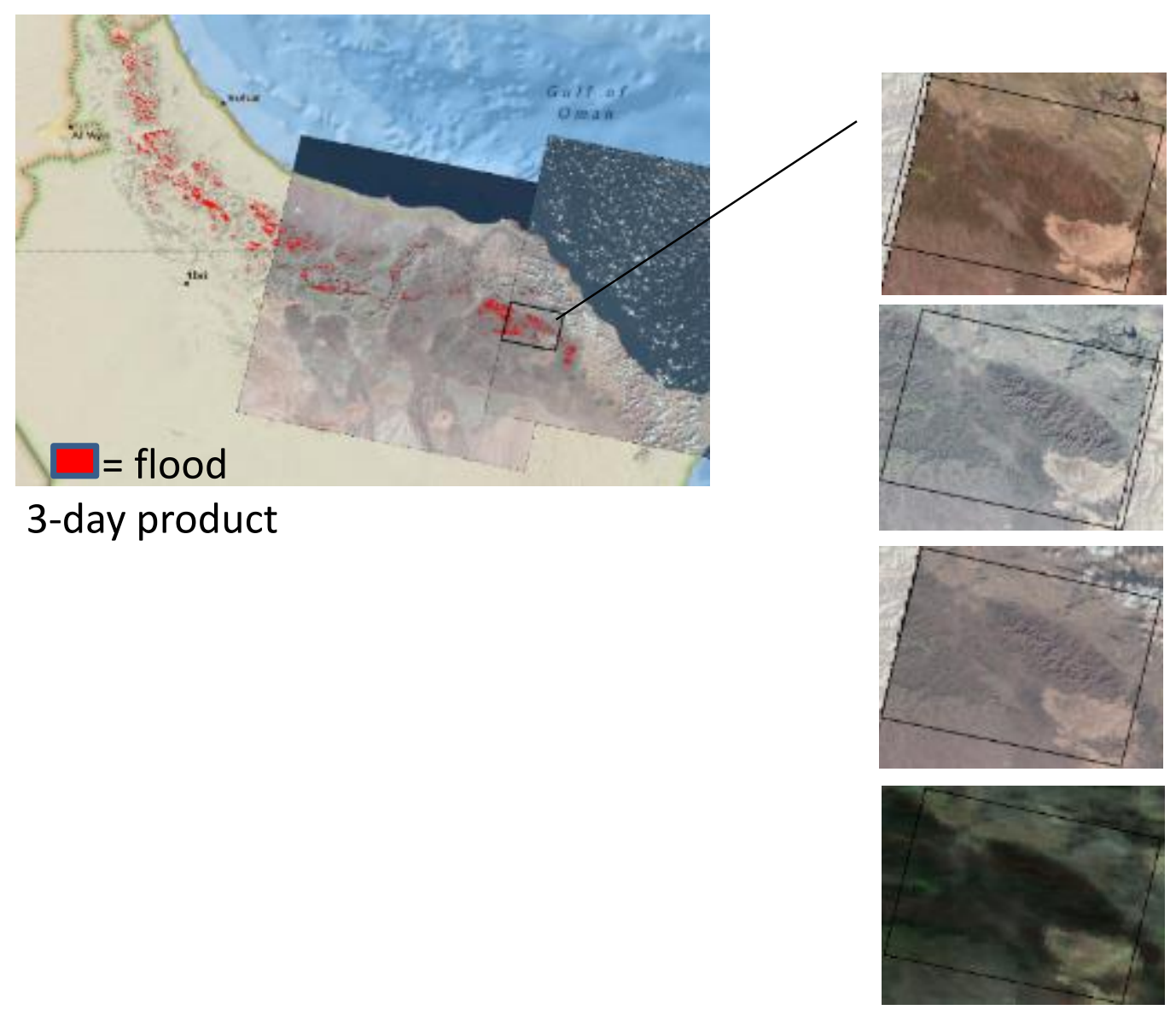

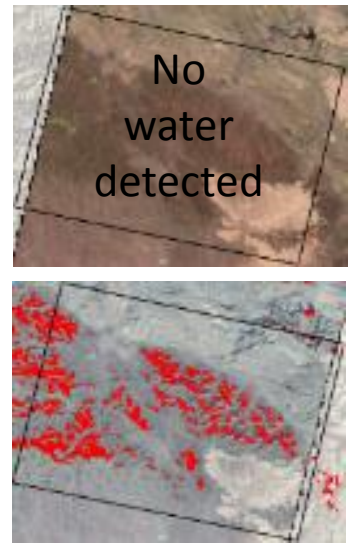

Landsat 8 June 17, 2013 (2013178)

Landsat 8 Nov 9, 2013 (2013313)

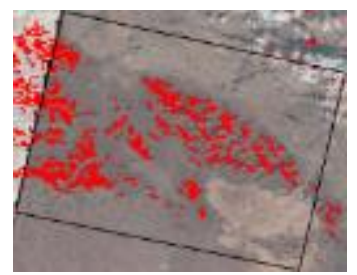

Landsat 8 Nov 18, 2013

(2013322)

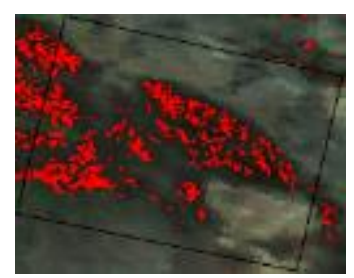

MODIS (MOD09) Nov 11, 2013 (2013315) 
Example: Barren rock / volcanic false positives

Mauna Loa, Hawaii: 17 Dec 2013

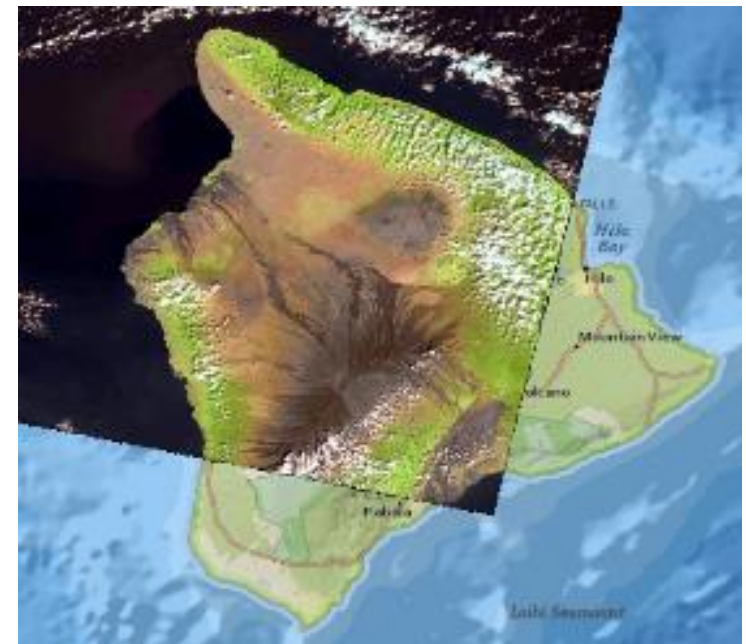

Landsat 8

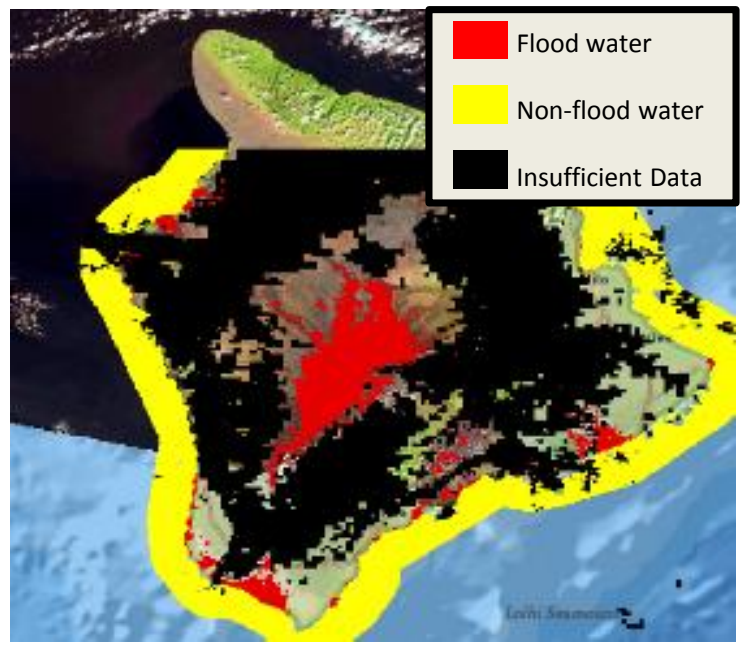

MODIS NRT Product 
Australia: 04 July 2014

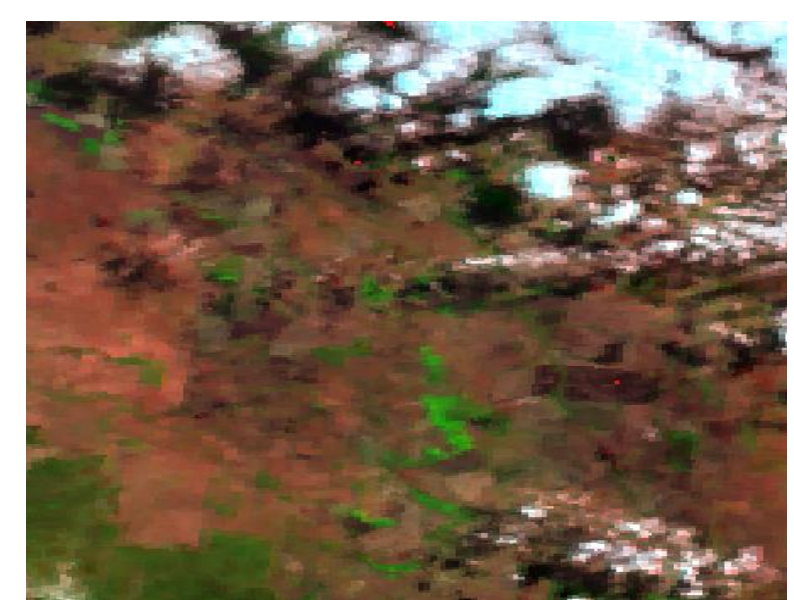

Input data: MOD09, 04 Jul 2014

3-day product removes most cloud shadow false positives

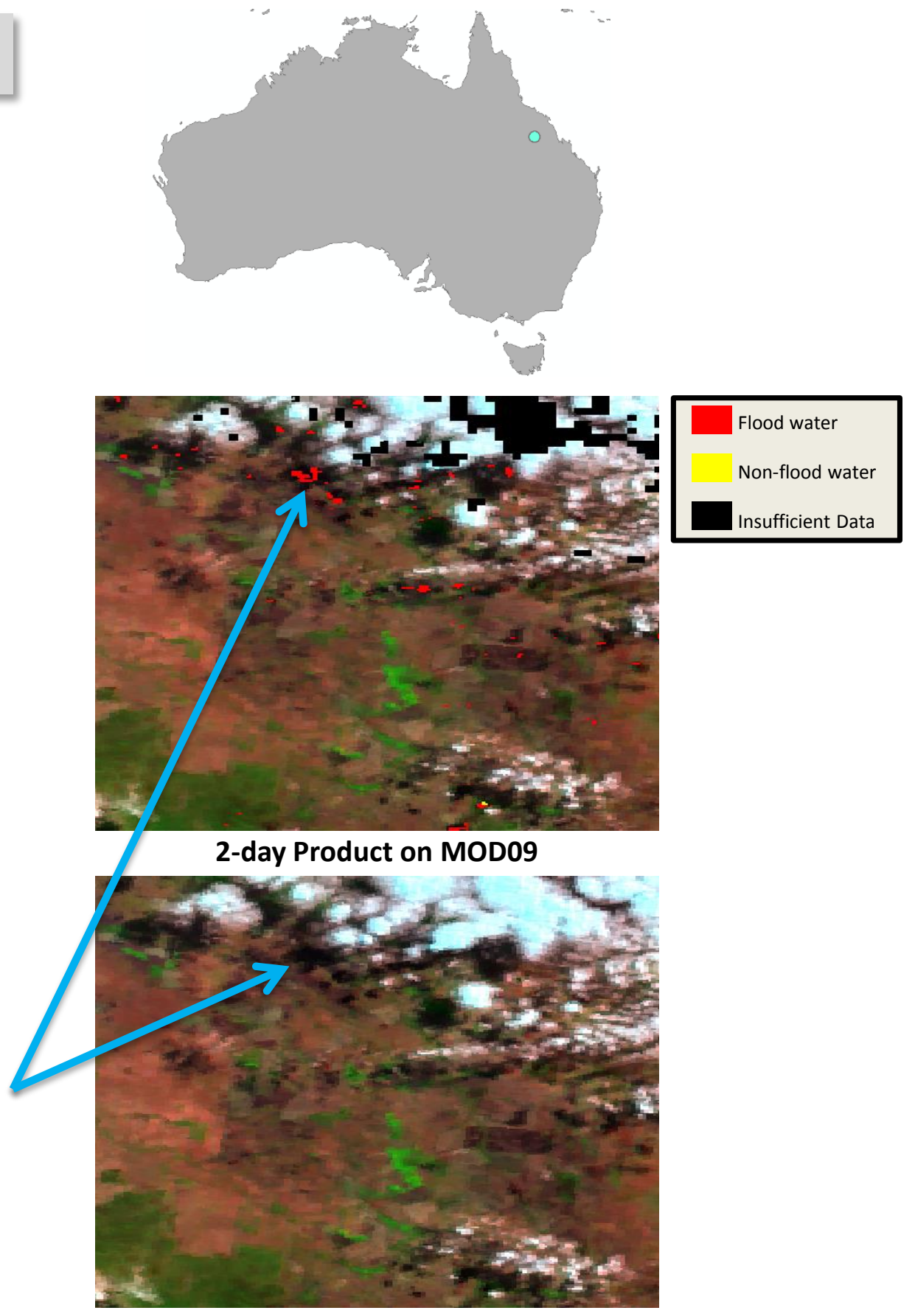


Comparison of different compositing periods: 2-day vs 3-day product

\section{Botswana: 24 Mar 2014}

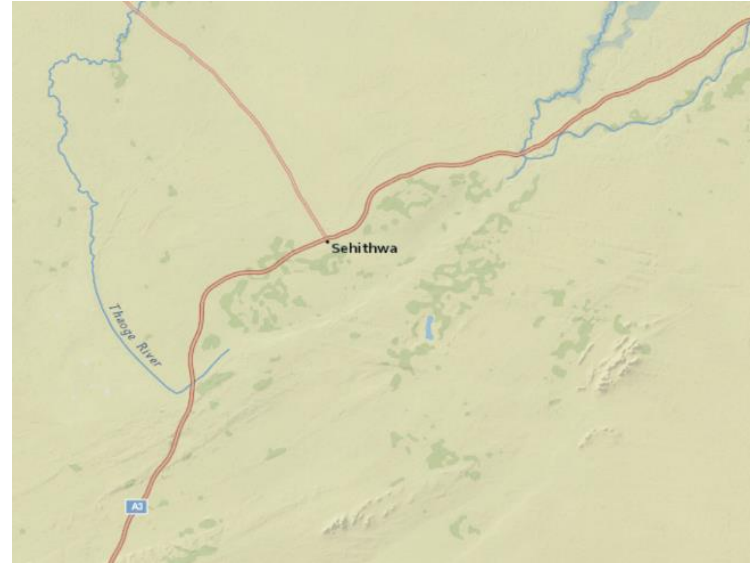

National Geographic base map

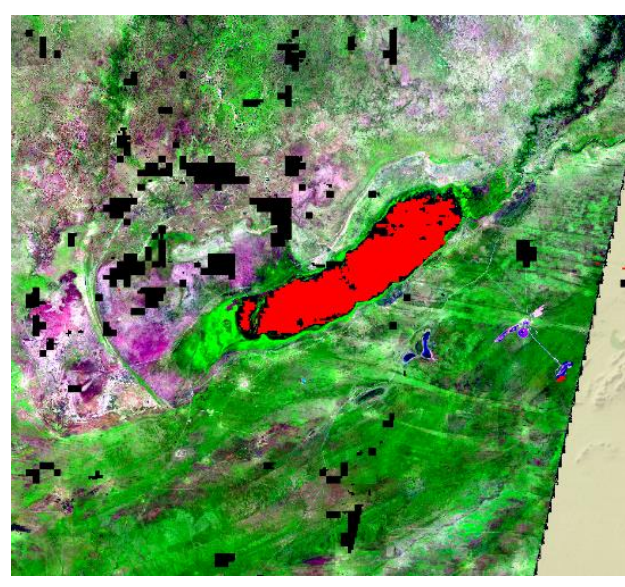

2-day product

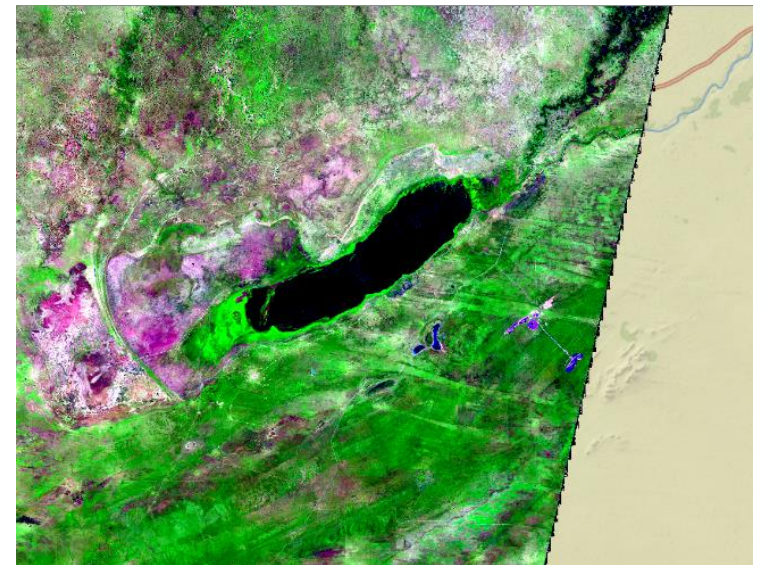

MODIS (MOD09) Mar 24, 2014

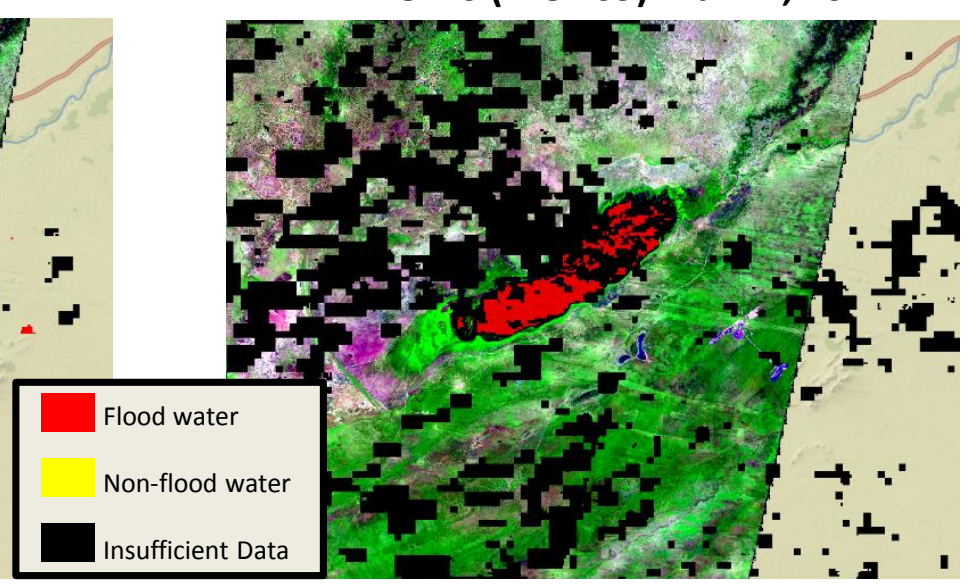

3-day product

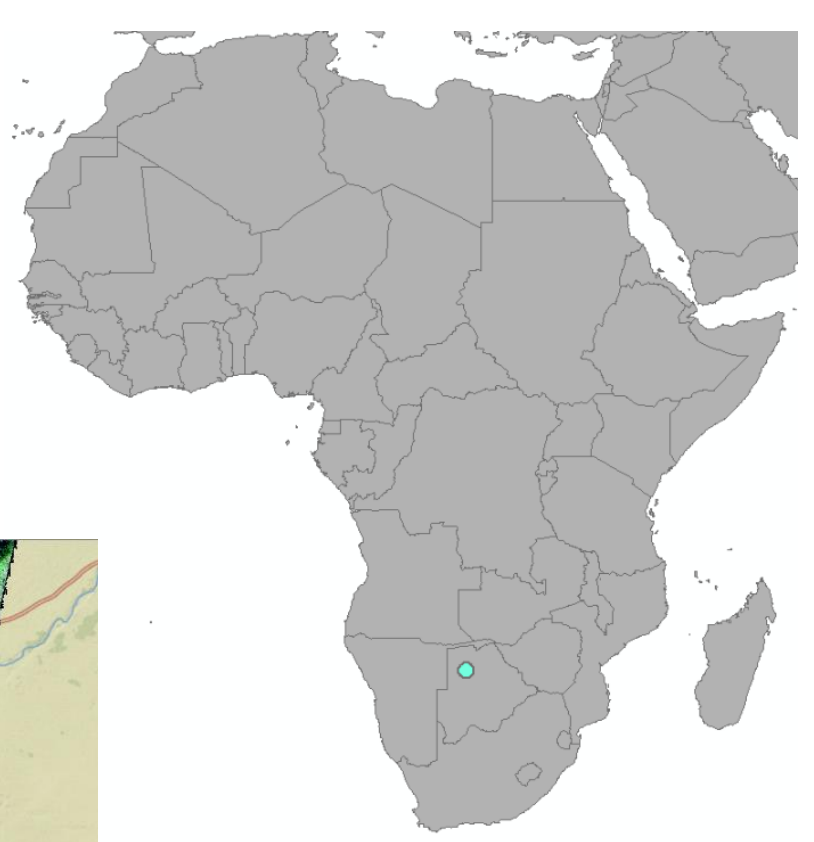




\section{Which compositing period should I use? Just how cloudy is it?}

- It depends....basically on cloud conditions:

- User tolerance for false positives (and false negatives)

- User need for only the most up-to-date information

- Clear conditions? Use 2-day or 1-day.

- Very sensitive to false-positives? Use 3-day.

- Very sensitive to false-negatives (cloud)? Use 14-day.

- Need the latest info? Use 1-day.

- Best approach? Look at them all and evaluate for given event and needs. 


\section{Current efforts}

- Recently transitioned flood map distribution to NASA LANCE

- Working transition of flood map production to NASA LANCE

- Improvements to MODIS product

- Replace $10^{\circ} \times 10^{\circ}$ Tiles with swath data

- Decreased latency

- Improved masking of cloud and terrain shadows

- Masking of high slope areas (HAND algorithm)

- Ephemeral water mask (recurring water that is not unusual flooding) 


\section{Comments/ Questions?}

